\title{
"Because Here, White is Right": Mental Health Experiences of International Graduate Students of Color from a Critical Race Perspective
}

\section{S Anandavalli ${ }^{1,2} \cdot$ L. DiAnne Borders ${ }^{2} \cdot$ Lori E. Kniffin ${ }^{4,3}$}

Accepted: 22 April 2021 / Published online: 24 May 2021

(c) The Author(s), under exclusive licence to Springer Science+Business Media, LLC, part of Springer Nature 2021

\begin{abstract}
To examine the mental health experiences of international graduate students of Color (IGSC) as they navigate through a multitude of systemic barriers, the researchers interviewed eight IGSC in the U.S. Adopting a critical race perspective, the researchers sought to address a persistent gap in the counseling literature, and explore how systemic influences of racism, xenophobia, and discriminatory policies impacted the mental health of IGSC. Three distinct themes emerged from phenomenological analysis of the participants' interviews in the current study: cross-cultural challenges, surviving racism and anti-immigrant sentiment, and mental health and wellness. Implications for how professional counselors can support IGSC as they navigate through multiple challenges are emphasized.
\end{abstract}

Keywords International graduate students of Color - Critical race theory · Interpretive phenomenological analysis $\cdot$ Social justice $\cdot$ Minority mental health

\section{Introduction}

International graduate students of Color (IGSC) are exposed to a multitude of interpersonal and systemic stressors that frequently go underrecognized in the counseling literature (Anandavalli, 2020). Extant scholarship indicated that, as a result of their

\section{S Anandavalli}

anandavas@sou.edu

1 Present Address: Clinical Mental Health Counseling, Southern Oregon University, 1250 Siskiyou Blvd, Ashland, OR 97520, USA

2 Counseling and Educational Development, The University of North Carolina at Greensboro, 1400 Spring Garden Street, Greensboro, NC 27412, USA

3 Department of Educational Leadership and Cultural Foundations, The University of North Carolina at Greensboro, 1400 Spring Garden Street, NC 27412 Greensboro, USA

4 Present Address: Leadership Studies Fort, Hays State University, Hays, KS, USA 
racial identities and immigration status, IGSC are frequent targets of explicit violence (Lee \& Rice, 2007), racial slurs (Dobinson \& Mercieca, 2020), and nativist rhetoric (Yao et al., 2020) within and outside of college campuses. Such incidents contributed to the significantly poorer mental health outcomes of international students of Color (ISC) compared to the American public (Ogunsanya et al., 2018). Since this inquiry, the novel coronavirus (COVID-19) has potentially further exacerbated entrenched xenophobia in the American society. Chirikov and Soria (2020) surveyed over 30,000 international students navigating the pandemic reality in the United States (U.S.). They noted that over 17\% of international undergraduate students and $12 \%$ of graduate students had been victims of intimidating, aggressive, or hateful behavior based on their nationality. Regrettably, the rate is higher (22-30\%) among international students from China, South Korea, Japan, and Vietnam, given pervasive Sinophobia in the U.S. (Chirikov \& Soria, 2020). The researchers found that, in addition to threatening students' safety, such incidents also had deleterious effects on the participants' mental health.

In addition to racism and nativism, IGSC also navigate professional and academic stressors, such as conducting research, developing strong publication potential, and teaching duties (George Mwangi, 2019). Often, their professional development is impeded by discriminatory visa restrictions that narrow down their funding and career opportunities. For instance, extant regulations prohibit Chinese international students from securing student visas for longer than five years (Federal Register, 2020) despite advanced doctoral work often demanding longer training timelines. Similarly, international students are prohibited from securing any employment outside of campus during their student status (United States Immigration and Customs Enforcement (USICE), 2020), except under dire circumstances deemed appropriate by the USICE (USICE, 2020).

Although existing counseling literature offers data on the stressors experienced by international students in general (e.g., isolation, limited English speaking skills), much of the literature on their mental health and educational experiences remain assimilationist in nature, wherein individual international students are recommended to adopt White/Western/American norms to maintain optimal health (Al-Krenawi et al., 2020), with inequitable social institutions rarely held accountable for inadequate and biased support systems (e.g., English exclusive university library staff and classrooms, visa policies). Critical race theory (CRT), however, offers a framework for an epistemic counternarrative (Bells, 1992; Delgado, 1995) from the perspective of the marginalized community - IGSC in this study. Thus, the purpose of this study was to adopt a CRT framework, and examine the mental health challenges of IGSC. Specifically, we sought to examine if systemic influences had a direct and ongoing impact on the psychological well-being of IGSC in the U.S. 


\section{Literature Review}

\section{Critical Race Theory}

Critical race theory positions the acknowledgement and countering of embedded racism as central to its framework. CRT scholars identified that racism is a normalized and everyday aspect of American society, wherein individuals' well-being is sharply influenced by their race (Bells, 1992; Delgado, 1995). In other words, CRT draws attention to the tacit but ongoing impact of racism on individuals', particularly on minoritized communities' everyday life. Additionally, CRT scholars noted that ignoring or minimizing the pervasive impact of racism on the lived experiences of persons of Color perpetuates color-neutrality (Yosso et al., 2009).

Several CRT scholars, such as Delgado (1995) and Bell (1992), noted that counternarratives and the larger analytical standpoint of CRT are often grounded in the experiential knowledge of minoritized communities who have a shared history of being othered. Thus, the othered accounts offer alternative perspective to hegemonic narratives of blaming minoritized communities for their distress, wherein communities hold inequitable institutions and social norms accountable for their pervasive harm (Delgado, 1995). For example, Xu (2020) shared that Chinese international students like herself are frequently expected to adopt Anglicized names, for the convenience of the dominant communities. Although the change may appear innocuous, the author argued that the name change is often symbolic of larger expectations to assimilate to U.S. White culture and shun one's cultural legacy. A CRT approach offers a viable framework to examine similar counternarratives among IGSC as they make meaning of their mental health experiences.

Furthermore, to effectively understand IGSC' lived experiences, one must consider their intersecting identities. CRT scholar Crenshaw (1989) observed that the experience of oppression is often intersectional, where identities stemming from race, gender, ethnicity, social class, and ability status have a compounding impact on the individual. Thus, CRT researchers strive for social justice that cuts across constructs of gender, race, social class, and ability status. By ignoring or being unaware of the profound role of systemic influences and barriers on IGSCs' mental health, professional counselors may fail to uphold the American Counseling Association (ACA) Code of Ethics that requires them to promote social justice (ACA, 2014). The Code of Ethics defines social justice as "the promotion of equity for all people and groups for the purpose of ending oppression and injustice affecting clients, students, counselors, families, communities, schools, workplaces, governments, and other social and institutional systems" (ACA, 2014, p. 3). The objective of this study was to address the gap in existing counseling literature on international students that offers limited emphasis on the role of systemic influences. Thus, to enhance professional counselors' knowledge various systemic influences on IGSC' mental health, we positioned CRT as a central framework in this inquiry. 


\section{International Graduate Students of Color}

According to the Council of Graduate Schools (CGS), international students in the U.S. constituted over 20\% of new enrollment in Fall 2019 (CGS, 2020). Furthermore, the proportion was significantly higher in STEM programs, such as computer science and engineering programs, wherein international students comprised more than half the enrollment (CGS, 2020).

Although study abroad programs can be an exciting experience, international students in graduate programs face multiple challenges, including demanding academic and research responsibilities that jeopardize their mental health (George Mwangi, 2019). Recently, Lin (2020) analyzed the experiences of five international doctoral students of Color using poetic analysis. A South Korean participant in her study, Sam, noted that, despite being an ambitious and competitive individual, he struggled throughout graduate school (Lin, 2020). He reported frustration as he could not effectively present his research in public, due to his English-speaking skills, and thus share his expertise with other scholars in the field. Eventually, he began to isolate himself during conferences and social events, due to his embarrassment and shame centering his accent and language competency. His professional development was further obstructed by scholarships and fellowships that were made available exclusively for domestic students, rendering limited opportunities for him-and international graduate students - to apply and secure grants and scholarships (Lin, 2020). Unfortunately, visa regulations pose another layer of challenge for international graduate students. These regulations require them to maintain full-time enrollment (a minimum of 9 graduate semester credits) and a 3.0 GPA, failing which they can be deported to their home country (USICE, 2020).

As highlighted by Crenshaw (1989), the lived experiences of individuals are rarely one-dimensional and are often impacted by the intersecting cultural identities, including race and citizenship status. Lee and Rice (2007) reported that one in four IGSC participants in their study had experienced an intense form of neo-racism from advisors, domestic students, faculty members, or members from the community. Participants shared that the pervasiveness of explicit hate crimes eventually became a normalized outcome of being an international student of Color. One student in the study shared, "Yeah, we generally walk back home from campus, and it was not a big deal, but people threw bottles at us. Being international students, you get used to it" (Lee \& Rice, 2007, p. 29).

Yao et al. (2020) explored the lived experiences of ISC after a racialized incident on campus, wherein a White identifying college student posted a video on YouTube stating, "I am the most active white nationalist in [the state]." Participants shared that they were really scared and found themselves in a constant state of hypervigilance, fearing they might encounter this individual on their college campus. The fear also prevented them from interacting with White identifying individuals for several months after the incident.

Although explicit forms of racism have a notable traumatic impact on IGSC (Anandavalli, 2020), scholars have noted that subtle and tacit forms of racism are equally harmful to students' mental health (Sue et al., 2019). For instance, linguistic 
racism (or the practices that conform, normalize, and perpetuate unequal power among language users Dovchin, 2020), has been used as a vehicle against ISC to further dehumanize them. Dobinson and Mercieca (2020) noted that, on an Australian university campus, international students were expressly prohibited from using their native languages and required to communicate in English. The scholars argued that such measures award inequitable privilege to English speakers due to their subjective linguistic capital (Dobinson \& Mercieca, 2020). Regrettably, international students have reported similar instances of linguistic racism within the U.S. as well (Lin, 2020; Mervosh, 2019).

Unfortunately, ISC also are targets of anti-immigrant sentiment cutting across partisan lines. In a recent speech, (December $9^{\text {th }}, 2020$ ), Secretary of State Michael Pompeo referred to Chinese international students with the racist trope "yellow peril." He called on U.S. colleges and universities to limit the number of Chinese international students on their campus, perpetuating Sinophobic sentiment in the community (United States Department of State, 2020). His rhetoric of labelling hundreds of thousands of Chinese international students as potential spies further reiterated a larger anti-immigrant and anti-Chinese sentiment in the U.S.

Regrettably, a combination of linguistic racism, xenophobia, and navigating through new cultural norms in the host country, often pushes IGSC into isolation and chronic loneliness. Girmay and Singh's (2019) participants reported rituals and practices that enhanced social connections in their home countries (e.g., meal times) were marked with individualism and independence in the U.S., resulting in painful solitude. The challenges of IGSC in particular, and international students in general, are further exacerbated as few counselors are adequately knowledgeable and culturally competent to work with this population (Liu et al., 2020; Nilsson et al., 2004). Due to this factor, among other reasons (e.g., stigma against counseling, students' limited knowledge of counseling services) international students' utilization of counseling services remain at $2 \%$ (Nilsson et al., 2004).

Thus, the objective of this inquiry was to advance counselors' and researchers' knowledge by examining the role of racism, xenophobia, and discriminatory policies in influencing the mental health experiences of IGSC - a persistent gap in the counseling literature. One research question guided this interpretive phenomenological inquiry: "How do systemic influences of racism, xenophobia, and discriminatory U.S. policies impact the mental health experiences of IGSC?".

\section{Method}

\section{Interpretive Phenomenological Analysis}

The present inquiry was part of a larger study exploring the lived experiences of IGSC in the U.S., conducted in late 2018 and early 2019. During this context, antiimmigrant, and anti-Black sentiment were significant. As discussed above, several anti-international student policies, and statements were encouraged under the then President's leadership. To gain detailed narratives of the experiences of IGSC, and create a platform for counter storytelling (Bell, 1992; Delgado, 1995), we adopted 
an Interpretive Phenomenological Approach (IPA; Smith, 1996). The primary focus of IPA is to examine how individuals make meaning of their lived experiences, and limit chances of researchers imposing their subjective beliefs and assumptions on the participants' experiences (Smith, 1996). The steps followed in this inquiry are discussed below.

\section{Researcher Roles and Trustworthiness}

There are four aspects of effective qualitative research (Guba, 1981), and each aspect was pursued by the team through diverse strategies: credibility (i.e., member checking, two coders), transferability (e.g., multiple data points for each subtheme), dependability (e.g., utilizing external auditor's feedback), and confirmability (e.g., field notes). At the time of the IPA inquiry (early 2019), the first author identified as an IGSC from India. The second author was a White American counselor educator who had experiences working with international students as a supervisor, educator, and consultant. The third researcher identified as a White American who has collaborated with international students in her former professional roles. Each researchers' reflexivity documentation, an integral part of IPA, served to identify potential blind-spots centering around typecasts and assumptions surrounding IGSC, and the role of institutions and structures on their mental health. The first author immersed herself in the IGSC community in a Southeastern city in the U.S. The cultural immersion and community building effort allowed for the research question for the larger study to emerge from community-based reflections with the participating community.

To examine the interviewer's biases, and scope for potential influence, two mock interviews with eligible IGSC were completed and recorded. Feedback from the volunteers and the co-authors was sought on the interview approach. No changes to interview approach were suggested by pilot study participants and the co-authors.

\section{Participants}

For this Institutional Review Board (IRB) approved study, the inclusion criteria were the following: participants needed to be at least 18 years of age; experienced in English (interview language); hold a valid F1 or J1 visa (higher education/student visas in the U.S.); and actively enrolled in any graduate program in the U.S. They were also required to self-identify as a Person of Color and should have lived in the U.S. for not longer than two years, given the evolving effects of cultural adjustment in the U.S. Recruitment flyers included information on the aim of the study and contact information for the first author. The flyers were shared through social media and via communication with various international students' offices at regional universities and colleges. Using convenience and snowball sampling, a total of 23 participants responded to the recruitment announcement. However, 12 did not meet inclusion criteria (e.g., identified as immigrant or refugees but not as international graduate students with F1 visa) and three did not respond until several months after the completion of data analysis (due to time constraints on the prospective interviewees' part). Eight individuals were deemed 
Table 1 Participant Demographics

\begin{tabular}{llllll}
\hline Pseudonym & Race & Age & Gender & Nationality & $\begin{array}{l}\text { Coun- } \\
\text { seling } \\
\text { Services }\end{array}$ \\
\hline M. F & Asian & 44 & Female & British & No \\
A. Z & Latina & 31 & Female & Brazilian & Yes \\
Bansal & Asian & 25 & Female & Indian & No \\
Jay & Asian & 30 & Female & Indian & No \\
K. S & Asian & 29 & Female & Chinese & Yes \\
Cheryl & Asian & 27 & Female & Indian & No \\
T. L & Asian & 25 & Female & Chinese & No \\
Fani & Asian & 33 & Female & Indonesian & No \\
\hline
\end{tabular}

eligible. They reviewed the IRB approved consent form before completing the individual, semi-structured interview and selected their own pseudonym. Participants' demographics and pseudonyms are available in Table 1. On completion of the interview, each participant received a \$10 gift card. Although the researchers did not screen for gender or sex, all participants in the study happened to identify as cisgender females.

\section{Data Collection}

Prior to the audio-recorded interview, each potential participant completed the consent form and an online demographic questionnaire to examine their eligibility. The interview schedule was developed by the first and second authors after critically analyzing the counseling literature on IGSC mental health. For instance, given limited research on IGSC' mental health, and the impact of racism on the same, interview questions were appropriately framed ("How did the early transition period affect your mood and wellbeing?"; "What was it like being an international graduate student of Color, experiencing challenges in a foreign country?"). Feedback on the interview schedule was sought from voluntary mock interviews completed with two eligible IGSC, and no changes were requested. Using the interview schedule, the first author interviewed eligible participants. The open-ended interview questions gave participants the opportunity to describe stressful and overwhelming experiences. Follow up questions (e.g., "How did racism and xenophobia impact your role as a student?") were also posed. The first author conducted and transcribed all interview recordings. Three interviews were conducted online and five were completed in-person at a location of each participant's choice. Interviews ranged from 60-120 min in length ( $M=72 \mathrm{~min})$. The first-author documented participants' presentation during the interview (e.g., body language, tears) to gain deeper understanding of their narratives. 


\section{Data Analysis}

To carefully account for the interviewer's subjectivity in data collection and analysis, all steps and field notes were documented in the reflexivity journal and made available to the co-authors. Among many conversations on researcher bias, dialogue on the first author's economic privilege and unearned access to financial resources before migrating to the U.S. is one example. In such instances of potential biases, the authors documented their lived experiences of privilege and oppression and consequent worldview, and explicitly considered these while analyzing the interviews.

The analysis team was comprised of two coders (first and third authors) and an auditor (second author). Each interview transcript was considered a unit of analysis. The coders read the first transcript several times to become accustomed to the material. In line with Smith and Osborn's (2007) recommendations, an open-coding protocol was followed. Similar themes within one participant's narrative were then grouped together to construct super-ordinate themes. Then, through consensus, coders developed a shared list of super-ordinate themes for the first participant's transcript. They followed the same procedure for the remaining seven participants. After coding all the individual interviews, the first and third authors created a grand theme list, based on all the participants' super-ordinate themes. The second author served as auditor to provide feedback on the representativeness of the themes. The grand theme list reflected the central experiences of the eight IGSCs. Respective individual and grand themes were shared with each participant to receive relevant feedback (member checking), with all participants responding that the themes were accurately reflective of their experiences. No changes were requested by the participants.

\section{Findings}

Three grand themes, with related subthemes, emerged from the interviews with the eight IGSC participants: (1) cross-cultural challenges, (2) surviving racism and anti-immigrant sentiment, and (3) mental health and wellness. These themes are presented below, supported by participants' quotes to elucidate their relevant experiences.

\section{Cross-cultural Challenges}

In this first grand theme, all eight participants described the multiple challenges they encountered as a result of transitioning into a new culture. They shared narratives of orienting themselves to a new culture, academic system, and social norms. Three subthemes were identified: financial constraints, isolation, and academic pressure.

The first subtheme was acute financial constraints. The participants reported that the exchange rate between U. S. dollar and their home currency posed significant financial limitations and stress. Bansal shared that she "just couldn't buy anything" for the first few months, and often depended on her parents in India and high interest 
rate education loans to navigate her study abroad experience. Jay noted that her fear of running out of money was so strong that she would isolate herself in her room:

"I spent very little money in the initial months and second guessed every purchase, even food. I hid in my room and did not want to go out anywhere, because it would need money."

A. Z. narrated that international students in her doctoral program were not offered funding for the first year: "International students in $\mathrm{Ph}$. D. here don't receive their stipend in the first year. So, on top of having doctoral level classes, a new life, and finding a new lab, I had to figure out how to get money." A. Z. disclosed that, given the financial constraints, she was "always thinking about saving money."

As participants transitioned into the host country, six participants also reported experiences of chronic isolation. Thus, the second subtheme was isolation. Fani, shared that it was "really hard to find friends in the U.S." Jay described her initial months in the U.S. as some of her most challenging in her life.

Initially I didn't feel much excitement. It was loneliness and stress. One after the other. That phase is so difficult. I needed to find people, someone who can guide me. Just talk to me. It was so bad. I was not able to enjoy... anything.

A. Z., who worked in a STEM research lab, often found herself alone and with no social contact outside of her lab.

In the lab ... no one talks to each other, they are cold and distant, and just see each other as competition. They are not curious ... So when I come home from lab, my little apartment is quiet. Nothing. In the starting days I felt like I have no one to talk to. Nobody knows what I am going through.

However, one participant M. F., a mother of two daughters, differed from the other seven participants. Having lived abroad before, she shared that she was prepared for the challenge.

You know it is going to be you... and only you. So when you have travelled around the world ... volunteered in various places ... I knew coming here that I would start from scratch. So it just wasn't that big a deal being alone.

The third subtheme was academic pressure. The participants observed that the academic culture in the U.S. was unique and required them to alter their existing notions of work-life balance and achievement. K. S., a doctoral student in biomedical engineering shared that she was chronically worried about her research agenda.

When I came to the U.S., my (American) classmates all started to work on their research. I was not prepared. I spent a lot of time trying to find a suitable topic. ...In the U.S. nobody guides you, it is not like China. So I have to go to each professor one by one and ask. I was so worried all the time. I would call my mother and cry on phone. 
Fani, a student in an MBA program, was stressed that if she did not do well in each of her courses her scholarship funding could be withdrawn, leaving her stranded mid-way in her graduate studies.

In my first semester here, I had so much stress. I got a very bad score in my midterms, and I was stressed. Panicked. Because I could lose my Fulbright scholarship and be sent home. I was so worried that I should never get another $C$ grade, and did not rest until I got my grades higher.

Bansal's admission to graduate school was conditional, so she had to secure high scores in her first semester to justify her eligibility for the graduate program of her choice. This meant that a lot was at stake in her first term academic performance.

The first semester was crucial for me. If I did not get a certain grade in all those courses, it meant I could be sent back, and all this money, this effort, time... wasted forever. So every time an exam came up, I was really scared. Nobody understood this situation. And nobody helps, because everyone views each other as a competition here.

\section{Surviving Racism and Anti-immigrant Sentiment}

Participants shared that they were frequent targets of direct and indirect racism and xenophobia. They reported experiences of being othered and noticed they were often perceived as unwanted in the U.S. Three subthemes were identified: linguistic racism, climate of fear, and xenophobia.

Linguistic racism refers to privileged positioning of one language over others, thus creating an inequitable environment to express complex thoughts. IGSCs found linguistic racism was a frequent experience. Three participants shared that they often felt "overwhelmed" by the extensive and exclusive usage of English in the U.S. They found themselves yearning for opportunities to engage in their native languages and experience a sense of familiarity. A.Z, who was raised in multilingual education (English and Portuguese) in her home country Brazil, missed speaking in Portuguese.

Ifelt overwhelmed in the beginning. I had classes in English, of course. I go to the grocery store, and they speak English. I turn on the TV, its English. I just want to hear something in my language (Portuguese) for one minute, but that is not possible. There is no room for anything. Hundred percent English!

Linguistic stress and exclusive use of English often prevented participants from engaging in deeper conversations and meaningful participation in classroom discussion. T. L. who was from China, felt like the language barrier had turned into an "intelligence barrier." 
Americans speak so fast... Even though they say it's ok, I feel dumb. I feel less than others, and the language has been made to feel like it is your intelligence barrier. I really want to say something in class... but I cannot say a word.

Climate of fear referred to a larger atmosphere of anti-immigrant sentiment experienced by the IGSC. Participants acknowledged that even when they were not direct targets of explicit forms of racism, they were hypervigilant about their safety. K. S., an IGSC from China, was deeply concerned that a Chinese international student was shot dead in the U.S. just months before she arrived in the country.

Before I came here, I was so worried about guns. Here everyone has one. Like a car. And to know that a Chinese girl was murdered here by a guy. I was afraid, and I guess it was a racist motive. It was scary.

K. S. shared that, because of incidents like this, she was often afraid to step out by herself. A. Z. also experienced a similar fear of stepping out of her apartment, especially after the 2016 Presidential elections: "I know I am an immigrant. And I look like one. So I was scared to go anywhere on my own after the elections ... The whole mood was of fear."

Cheryl too noticed that, when she arrived in the U.S., she was in a continual state of vigilance, and worried about who the next victim would be.

But when Trump got elected, I definitely felt like I could get deported any day, for any reason. And even if I don't get deported, I am experiencing lack of security and safety, because... you never know. So I had a huge reaction to that... In the U.S. you become a minority automatically. I could be brown, yellow, whatever. If I am not White... I am minority. And they make you very aware that you are not a citizen. That you are not White.

In the third subtheme, xenophobia, we included narratives of direct oppression and xenophobia the participants experienced as a result of their intersecting identities of race, nationality, and faith. M. F., a British-Indian, Catholic participant, often went to her daughter's soccer practice.

I don't know how it came up in conversation. She basically asked me what religion was I and I'd said I was Catholic and she said, "Oh, I thought you might be Hindu, but I'm so glad you are Catholic." And I said, why? "It would be really difficult for me if you are Hindu, if you believed in all those gods and you didn't believe in Jesus and you weren't Christian, that would really be difficult for me to be friends with you." I just couldn't believe it!

Cheryl shared that people around her were quick to Other her and communicate her outsider status in very clear ways. She recollected an incident when she had asked the university career counselor's help in finding documents and opportunities to help her Austrian partner migrate to the U.S.

Instead of helping me.. or refusing to help me... she simply told me to leave the country if I wanted to live with my partner. And then it hit me. Of course, I cried... It hit me that I am not wanted here. I am outsider here. 
Cheryl shared that racism was often covert, through gestures and differential treatments meted out to IGSC.

It is the subtle ones too... where my international friends, who are TA [teaching assistants], are criticized by students for their accents. Are judged for their way of teaching ... so to escape that ... I just code switch. I change my accent as soon as a White person or an American walks in.

Participants in the study felt their outsider status was often based on their racial identity, linguistic skills, and notions of Americanism. As A. Z. shared, "here (in the United States), White is ... right."

\section{Mental Health and Wellness}

IGSC in the study experienced a variety of mental health symptoms that ranged from mild symptoms of depression to suicidal ideation. They often battled these challenges on their own, fearing that no one would truly understand their experiences. Three subthemes were identified: acute mental illness symptoms, chronic symptoms, and counseling.

Participants experienced significant acute mental illness symptoms, often stemming from a combination of stressors. A. Z. shared that as a result of her academic stress and chronic homesickness, she experienced panic attacks. Eventually her mental health got worse; one night she experienced heightened suicidal ideation.

I was working every weekend during that time, and during the week I would work several hours and come home only to sleep. I was on a loop of working, and my advisor was still not happy. And one night, I thought of jumping out of the window. I cannot handle this anymore. I thought I will end this, and everything will be fine.

Eventually A. Z. went to her bathroom and prayed for her suicidal ideation to subside. Later in the interview she shared that she "thanked God for not having access to a roof that night."

Participants' experience of mental illness symptoms also impacted their physical health. Fani, a Muslim student away from her family in Indonesia, battled constant stress. One night she developed psychosomatic symptoms, and found one side of her body paralyzed.

I felt like I cannot move my body. I thought I got a stroke, but the ER doctor told me I was stressed. I was at a level of stress that I wasn't even aware of. I know that I missing my family, my kids, my husband, my mother. I know I have to work hard here. I know there is Islamophobia here. And finding a friend here is difficult. I think it all got together, and made me very stressed. 
Participants also coped with multiple chronic symptoms that developed after their arrival to the U.S. These longer-term symptoms included sadness, homesickness, and insomnia. T. L. shared that the initial months of coping with a new culture and new language was challenging for her. She found herself crying after her classes, and feeling helpless.

I would say the only thing that helped me then...crying. I would cry, and cry, and cry. Waiting for this phase to get over, until I can be ... a real person. Until people can understand what I speak. I can understand what they speak. I cried to my teachers. To my advisor. To my parents. To myself.

Bansal, who was refraining from sharing her struggles with anyone, noticed she had trouble sleeping. She shared that she rarely slept for longer than five hours a night, and "even that five hours was poor sleep." She also noted her motivation levels were low.

I would just stay in my room all day, if I didn't have classes. Not meet anyone. Not try anything new. It is like I didn't want to do anything that wasn't required. It was a difficult phase... when I had zero motivation. But of course, I cannot tell my parents back in India, because I know they will worry. I cannot tell anyone here, because I am not that close to anyone yet... so I just waited.

As participants navigated challenging stressors, they also discussed their specific help-seeking strategies and the rationale underlying their choices. Of the eight participants, only two participants sought counseling.

K. S. discontinued counseling after two sessions, as she didn't find the campus sessions to be helpful: "Actually I don't feel that helped... So when I went to the counseling service I was actually looking more for the actual advice instead of someone just listening to you. I wanted directions and resources." She also felt frustrated by the limited nature of individual counseling:

"And what I don't like is, every session is...was an hour. So you're just introduced something but the time is out. That's what I hated most about."

However, A. Z. found counseling supportive, and observed,

"You don't know anyone but I want to talk about what I think, what I want and what I'm thinking about. So that helped a lot. It was good. It was very helpful. Very helpful."

The remaining participants in the study disclosed that they knew little about the counseling center and the specific support the resource may provide. Jay noted,

"I don't know much about the campus counseling for services. ...I think that many do not know much about what counseling ... can do for the international students. “

Bansal agreed with Jay, and noted that few international students knew about counseling services. 
But I partly blame the university... never told us what counseling is, what it is for, and how I should access. Why did they not talk about it, especially when graduate school is filled with international students? A brief email reminding us of such service... is bare minimum... We are from a new culture and we often don't know about these things (campus counseling center).

\section{Discussion}

Extant mental health and higher education literature on international students has been predominantly assimilationist (George Mwangi et al., 2019), advising students to adapt to dominant U.S. norms as a means to alleviate their psychological distress. However, the CRT framework views distress of minoritized communities, including IGSC, as symptomatic of institutional barriers and limitations (e.g., limited institutional support for non-native English speakers, like T. L., may exacerbate their sense of isolation and academic pressure). Thus, this study advances existing counseling literature by contextualizing the mental health experiences of IGSC with clear reference racism, xenophobia, and discriminatory visa policies.

The research question for this study centered around how systemic influences of racism, xenophobia, and discriminatory policies impact the mental health of IGSC. Participants noted that they often felt disconnected in nativist, American, and English-centric classrooms and social spaces. They were frequently reminded of their "outsider" status in the country, heightening their sense of alienation. These findings align with extant scholarship demonstrating that IGSC are often exposed to detrimental levels of academic pressure as a result of their intersecting identities. Lin (2020) found that many IGSC are ineligible to seek external funding and feel limited by their English-speaking skills, consequently adding additional stress to their academic and professional development as emerging professionals. Although existing counseling scholars have explored homesickness experienced by international students (e.g., Al-Krenawi et al., 2020), an unambiguous reference to the role of larger American society in Othering and excluding IGSC in everyday experiences (e.g., Cheryl's experiences at career counseling center) is absent. A CRT framework, however, holds higher education institutions, and the dominant society accountable for their role in IGSC' mental health.

Relatedly, the IGSCs' academic stressors were not insular to the larger role of systemic xenophobia. Complex visa regulations (e.g., limited financial aid, deportation threats) posed a significant threat to their well-being. For instance, despite the high academic pressure, participants had to main their full-time enrollment status and a minimum of 3.0 GPA. Furthermore, they experienced poverty and food insecurity as a result of USICE rules limiting their sources of income (USICE, 2020), as IGSC often worked for low wages for a maximum of $20 \mathrm{~h}$ per week. As Jay described, students would often "hide" in their rooms fearing additional expenditure, exacerbating their isolation in a foreign country. George Mwangi et al. (2019) noted their participants reported similar experiences of fear, isolation, and financial insecurity as a result of biased visa rules. Similarly, in a recent inquiry by Lertora and Croffie (2019), international students in counseling programs accredited by Council for 
Accreditation for Counseling and Related Educational Programs (CACREP) noted that visa regulations posed a chronic concern to them, affecting their career decisions and mental health.

Language was another source of oppression for the IGSC. Although extant research inquiries in counseling focus on international students' English language proficiency ( $\mathrm{Li}$ et al., 2018), few examined how the dominance and hegemonic use of English limits IGSC' meaningful engagement in classrooms and community (Anandavalli, 2020). As Cheryl described, IGSC' English language skills and accents were used to delegitimize their presence and role, including their roles as teaching assistants. Scholars have highlighted that having individual international students singularly responsible for adapting to English-centric communities is unfair (Dovchin, 2020). Given that T. L. experienced significant distress due to Englishcentric classrooms, Critical scholars such as George Mwangi (2019) and Lin (2020) indicated that universities must be held responsible for inadequate infrastructure to prepare and accommodate for multilingual students. They noted that universities offer limited guidelines for faculty and staff interacting with IGSC, all of which contribute to significant distress for students like T. L. and K. S.

Participants indicated the large-scale sentiment of xenophobia often percolated into community-level interactions. In other words, the actions of community members towards the participants were not immune to the actions and statements of political leaders and governments towards groups of international students (e.g., Chinese international students, Muslims). Thus, domino effects of large-scale xenophobia were evident in many participants' narratives. Anandavalli and colleagues (2020) noted that, during uncertain sociopolitical contexts such as the Trump era and the COVID-19 prompted pandemic, minoritized international students are especially likely to be victimized for their racial identities. Furthermore, Yao et al. (2020) noted the profound effect of White supremacy and xenophobia on ISC, and the denigrating message universities may send when they take minimal action against antiimmigrant tropes.

Despite the challenges to their mental wellbeing, however, few participants sought the support of the counseling services. Only one participant engaged in long term counseling and found it to be helpful. Over half the participants in the present study knew little about counseling or the presence of the campus counseling center. Nilsson et al. (2004) found that only $2 \%$ of international students access campus counseling services, and over $38 \%$ percent drop out after the initial session. Unfortunately, most of the participants in the present study resorted to managing their distress on their own. In the context of the current findings, it is plausible that the low utilization rate of campus counseling services may be partly attributed to institutional failures in adequately publicizing the multiple resources offered. Furthermore, as K. S. disclosed, counselors may have to engage in culturally responsive practice, as the needs of IGSC are not comparable to that of domestic students. 


\section{Implications for Professional Counselors}

All participants experienced profound mental health challenges that frequently resulted in psychosomatic symptoms and mood changes. This inquiry offers several implications for professional counselors currently or intending to work with IGSC. First, all participants observed direct experiences of racism, and nativism had a significant impact on their perceived safety and psychological well-being. Thus, professional counselors must acknowledge the role of systemic racism and xenophobic policies that have a direct impact on the deteriorating mental health of IGSC. It seems imperative that professional counselors be trained to explicitly address the deleterious impact of systemic racism and xenophobia on the lived experiences of all ISCs, including IGSCs (Sue et al., 2019). Second, to disrupt the isolation that IGSC encounter during their stay in the U.S., we recommend that community and college counselors engage in proactive mental and physical health outreach programs. Given the widespread stigma centering mental illness in various cultures, college counselors could organize mental health workshops in destigmatizing spaces such as the university recreation center and student lounges. Similarly, community counselors can set up mental health screenings at local libraries, places of worship, and recreation centers, especially during holiday seasons and periods of political instability, to effectively support IGSC.

Third, extant literature, as well as participant K. S.' experiences with counseling, indicate that many international students tend to discontinue the service prematurely, as goals and cultural expectations of counseling vary for this community (Nilsson et al., 2004). Broaching can serve as an effective tool for professional counselors to examine how their own and their IGSC clients' cultural identities impact their worldviews, goals in counseling, and experiences of privilege and marginalization (Day-Vines et al., 2018). Through transparent and culturally responsive dialogues on IGSC clients' mental health experiences, counselors can make efforts to reduce premature termination of counseling in this community. Broaching can also help counselors observe potential stigma against mental health, and internalized Whiteness among IGSC clients. Fourth, participants divulged that multiple restrictive visa policies impeded their access to resources. Discriminatory visa policies limited financial resources for participants, prompting food insecurity in at least three individuals. Given counselors' commitment to issues of social justice and multiculturalism, as stated in the American Counseling Association Code of Ethics (ACA, 2014), counselors, especially college counselors, are urged to collaborate with university administration to initiate food drives, home supplies donation, and scholarship opportunities dedicated to international students to support their holistic well-being.

Lastly, counselors also serve as change agents and social justice advocates, per the multicultural and social justice counseling competencies (Ratts et al., 2015). Participants in the inquiry disclosed fear of hate speech and xenophobia-prompted harassment, similar to sentiments disclosed by other ISC in past research inquiries (Dovchin, 2020; George Mwangi, 2019). We encourage professional counselors to prioritize the mental health of IGSC and advocate against xenophobic acts perpetuated at the regional, state, and national level. 
Although the inquiry is predominantly aimed at guiding professional counselors working with IGSC, the current study holds implications for counselor educators as well. As the number of IGSC and immigrants in the U.S. continue to rise, it is very likely that visa issues, linguistic racism, and xenophobia continue to have a dramatic impact on society's mental health. Participants' narratives of acute psychological distress serve as an urgent reminder to actively incorporate immigrant and international persons' mental health into the counseling curriculum.

\section{Limitations}

In qualitative inquiries, given relatively smaller and more homogenous samples, transferability of findings to different settings should be based on counselors' and researchers' deep and contextualized knowledge. Although the present study offers practitioners and educators knowledge about the mental health experiences of IGSC, the study suffered from a few limitations. Firstly, the term Person of Color is often used as an umbrella term to denote the identities of non-White individuals. However, the specific racialized experiences of the participants were often based on nuanced racial and ethnic identities. For instance, the experiences of a Black, Congolese IGSC in the U.S. may vary significantly from a light skinned Brazilian IGSC, given the embedded colorism and anti-Blackness in the U.S. society. Due to the use of the broader identity of Person of Color, details on such specific experiences may have been missed in this inquiry. Secondly, although we explored the deleterious impact of linguistic racism on IGSCs' lived experiences, the data collection interviews were conducted in English due to limitations in the researchers' proficiency of non-English languages. The use of a non-native language could have limited the complex and vulnerable ideas the participants disclosed in the study. Lastly, the impact of a multitude of other intersecting identities such as gender, sexual orientation, and ability status were not explicitly central to the inquiry. Thus, practitioners and researchers should be cautious as they extend the findings of this study to other relevant groups.

\section{Future Directions}

Participants in this inquiry went through challenging experiences that resulted in dangerous health consequences. It was noted that participants often navigated their mental health challenges on their own. They felt isolated and disconnected from sources of potential help. Thus, researchers are encouraged to examine the help seeking behaviors of IGSC, specific to mental health. Although Nilsson et al. (2004) examined the campus counseling service utilization rate for international students in general, studies examining specific factors that impact help seeking behaviors of IGSC could prove beneficial. Additionally, IGSC participants experienced stressors that were a result of their academic status, immigration status, and racial identities. Thus, considering intentional consideration of systemic influences on the mental health of IGSC is a valuable perspective in counseling research. Researchers may wish to explore the psychological 
well-being of IGSC from a systemic trauma perspective. Also, professional counselors' preparedness in addressing these issues is unknown. Research studies examining the preparedness of counselors in attending to systemic issues encountered by IGSC could provide valuable data. Lastly, researchers gather data on specific psychological strengths (e.g., resilience) demonstrated by IGSC (Anandavalli, 2021). Knowledge of IGSC' strengths may aid in counselors effectively leveraging in them in counseling setting and actively incorporating them in treatment planning.

\section{References}

Al-Krenawi, A., Alotaibi, F., \& Elbedour, S. (2020). Acculturative stress among female Saudi college students in the United States. Community Mental Health Journal, 57, 372-379 APA PsycInfo®. https://doi.org/10.1007/s10597-020-00659-8

American Counseling Association. (2014). ACA code of ethics and standards of practice. https://www. counseling.org/Resources/aca-code-of-ethics.pdf

Anandavalli, S. (2020). Lived experiences of international graduate students of color and their cultural capital: A critical perspective [Doctoral dissertation, The University of North Carolina at Greensboro]. Dissertation Abstracts International Section A: Humanities and Social Sciences. https:// libres.uncg.edu/ir/uncg/f/Anandavalli_uncg_0154D_12696.pdf

Anandavalli, S. (2021). Strengths-based counseling with international students of color: A community cultural wealth approach. Journal of Asia Pacific Counseling, 11(1), 111-124. https://doi.org/10. 18401/2021.11.1.7

Anandavalli, S., Harrichand, J. J., \& Litam, S. D. A. (2020). Counseling international students in times of uncertainty: A critical feminist and bioecological approach. The Professional Counselor, 10(3), 365-375. https://doi.org/10.15241/sa.10.3.365

Bell, D. A. (1992). Faces at the bottom of the well: The permanence of racism. Basic Books.

Chirikov, I., \& Soria, K. M. (2020). International students' experiences and concerns during the pandemic. SERU Consortium, University of California - Berkeley and University of Minnesota. https:// docs.google.com/document/d/11Zdc4dWiX9C_8o1vH-4H7XokIHoJ4yjjVNRv0vS3tBE/edit\#headi ng=h.ivkygr983tef

Council of Graduate Schools. (2020). International Graduate Applications and Enrollment: Fall 2019. Washington, DC: Author. Retrieved 21st April 2020, from https://cgsnet.org/ckfinder/userfiles/files/ CGS\%20Fall\%202019\%20International\%20Report.pdf

Crenshaw, K. W. (1989). Demarginalizing the intersection of race and sex: A Black feminist critique of antidiscrimination doctrine, feminist theory and antiracist politics. University of Chicago Legal Forum, 140, 139-167. https://chicagounbound.uchicago.edu/uclf/vol1989/iss1/8

Day-Vines, N. L., Booker Ammah, B. B., Steen, S., \& Arnold, K. M. (2018). Getting comfortable with discomfort: Preparing counselor trainees to broach racial, ethnic, and cultural factors with clients during counseling. International Journal for the Advancement of Counselling, 40, 89-104. https:// doi.org/10.1007/s10447-017-9308-9

Delgado, R. (Ed.). (1995). Critical race theory: The cutting edge. Temple University Press.

Dobinson, T., \& Mercieca, P. (2020). Seeing things as they are, not just as we are: Investigating linguistic racism on an Australian university campus. International Journal of Bilingual Education and Bilingualism, 23(7), 789-803. https://doi.org/10.1080/13670050.2020.1724074

Dovchin, S. (2020). The psychological damages of linguistic racism and international students in Australia. International Journal of Bilingual Education and Bilingualism, 23(7), 804-818. https://doi. org/10.1080/13670050.2020.1759504

Federal Register (2020). Suspension of entry as nonimmigrants of certain students and researchers from the People's Republic of China. https://www.federalregister.gov/documents/2020/06/04/202012217 /suspension-of-entry-as-nonimmigrants-of-certain-students-and-researchers-from-the-peopl es-republic 
George Mwangi, C. A., Changamire, N., \& Mosselson, J. (2019). An intersectional understanding of African international graduate students' experiences in U.S. higher education. Journal of Diversity in Higher Education, 12(1), 52-64. APA PsycArticles®. https://doi.org/10.1037/dhe0000076

Girmay, M., \& Singh, G. K. (2019). Social isolation, loneliness, and mental and emotional well-being among international students in the United States. International Journal of Translational Medical Research and Public Health, 3(2), 75-82. https://doi.org/10.21106/ijtmrph.82

Guba, E. G. (1981). Criteria for assessing the trustworthiness of naturalistic inquiries. Educational Communication and Technology: A Journal of Theory, Research and Development, 29(2), 75. https://doi. org/10.1007/BF02766777

Lee, J. J., \& Rice, C. (2007). Welcome to America? International student perceptions of discrimination. Higher Education, 53(3), 381-409. https://doi.org/10.1007/s10734-005-4508-3

Li, C., Lu, J., Bernstein, B., \& Bang, N. M. (2018). Counseling self-efficacy of international counseling students in the U.S.: The impact of foreign language anxiety and acculturation. International Journal for the Advancement of Counselling, 40(3), 267-278. https://doi.org/10.1007/s10447-018-9325-3

Lin, H.-C. (2020). Second language, body, and race-A poetic analysis of international doctoral students' experiences at a university. International Journal of Qualitative Studies in Education. Advance online publication. https://doi.org/10.1080/09518398.2020.1783015

Liu, H., Wong, Y. J., Mitts, N. G., Li, P. F. J., \& Cheng, J. (2020). A phenomenological study of East Asian international students' experience of counseling. International Journal for the Advancement of Counselling, 42(3), 269-291. https://doi.org/10.1007/s10447-020-09399-6

Lertora, I. M., \& Croffie, A. L. (2019). The lived experiences of international students in a CACREP counseling program. International Journal for the Advancement of Counselling, 42, 174-190. https://doi.org/10.1007/s10447-019-09394-6

Mervosh, S. (2019). Duke University apologizes over professor's email asking Chinese students to speak English. The New York Times. https://www.nytimes.com/2019/01/27/us/megan-neely-duke-chinese.html

Nilsson, J. E., Berkel, L. A., Flores, L. Y., \& Lucas, M. S. (2004). Utilization rate and presenting concerns of international students at a university counseling center. Journal of College Student Psychotherapy, 19(2), 49-59. https://doi.org/10.1300/J035v19n02_05

Ogunsanya, M. E., Bamgbade, B. A., Thach, A. V., Sudhapalli, P., \& Rascati, K. L. (2018). Determinants of health-related quality of life in international graduate students. Currents in Pharmacy Teaching and Learning, 10(4), 413-422. https://doi.org/10.1016/j.cptl.2017.12.005

Ratts, M. J., Singh, A. A., Nassar-McMillan, S., Butler, S. K., \& McCullough, J. R. (2015). Multicultural and social justice counseling competencies. https://www.counseling.org/docs/default-source/compe tencies/multicultural-and-social-justice-counseling-competencies.pdf?sfvrsn=8573422c_20

Smith, J. A. (1996). Beyond the divide between cognition and discourse: Using Interpretative Phenomenological Analysis in health psychology. Psychology and Health, 11(2), 261-271

Smith, J. A., \& Osborn, M. (2007). Interpretive phenomenological analysis. In J. A. Smith (Ed.), Qualitative psychology: A practical guide to research methods (2nd ed., pp. 53-80). SAGE.

Sue, D. W., Alsaidi, S., Awad, M. N., Glaeser, E., Calle, C. Z., \& Mendez, N. (2019). Disarming racial microaggressions: Microintervention strategies for targets, White allies, and bystanders. American Psychologist, 74(1), 128-142. https://doi.org/10.1037/amp0000296

United States Department of State (2020). The Chinese Communist party on the American campus. https://www.state.gov/the-chinese-communist-party-on-the-american-campus/

United States Immigration and Customs Enforcement (2020). Employment. https://www.ice.gov/sevis/ employment

Xu, X. (2020). Exploring the logic of name changes and identity construction: A reflective self-narration of assimilation expectations. Names, 68(1), 32-41. https://doi.org/10.1080/00277738.2018.1452937

Yao, C. W., Briscoe, K. L., \& Rutt, J. N. (2020). In the aftermath of a racialized incident: Exploring international students of color's perceptions of campus racial climate. Journal of Diversity in Higher Education. Advance online publication. https://doi.org/10.1037/dhe0000179

Yosso, T. J., Smith, W. A., Ceja, M., \& Solórzano, D. G. (2009). Critical race theory, racial microaggressions, and campus racial climate for Latina/o undergraduates. Harvard Educational Review, 79(4), 659-690. https://doi.org/10.17763/haer.79.4.m6867014157m7071

Publisher's Note Springer Nature remains neutral with regard to jurisdictional claims in published maps and institutional affiliations. 